\title{
ICon: Interference Concentration for Uplink in MultiCell OFDMA Networks
}

\author{
Dorna Bandari*, Gregory Pottie*, Pascal Frossard ${ }^{\dagger}$ \\ *University of California, Los Angeles, USA \\ †École Polytechnique Fédérale de Lausanne (EPFL), Switzerland \\ Email: dorna@ucla.edu,pottie@icsl.ucla.edu,pascal.frossard@epfl.ch
}

\begin{abstract}
In this work we propose a novel inter-cell interference coordination (ICIC) and resource allocation method. Our aim is to maximize the rate of the worst performing user in all cells. We solve the problem in two phases, inter-cell and intracell resource management: first we define an ICIC scheme called interference concentration (ICon) in order to manage resources across cells, then each cell independently performs resource allocation to its users while meeting the constraints imposed by ICon. Finally, we adapt the ICIC method in order to balance the performance achieved in neighboring cells. Differently than Soft Frequency Reuse (SFR), ICon assigns received interference power limits, or the Interference Power Profiles (IPPs) rather than transmit power limits. The IPP determines the interference level the cell tolerates on each band. The intuition behind this change is that the interference to a given cell can be concentrated on a small band, resulting in more efficient use of bandwidth. In the intracell resource management phase, each cell allocates power and sub-bands to its users given their location in the cell, maximizing the minimum rate such that the IPP of none of its neighboring cells is violated. In order to balance the performance across all cells we use gradient-like updates to IPPs of cells. Finally we simulate an LTE-like system and compare the performance of our method with reuse 1 , static FFR and SFR with proportionally fair scheduling of users in each cell. Static ICon achieves $18 \%$ higher 5 percentile rate than reuse 1 which was the best of these methods. Adaptive ICon is found to converge almost immediately, and adds an additional $11 \%$ to this gain.
\end{abstract}

\section{INTRODUCTION}

The aim in this work is to allocate resources to users for uplink transmission in a 2-D multi-cell OFDMA network, maximizing minimum rate achieved by any user. The solution can be directly applied to improving cell edge user performance in OFDMA based networks such as Long Term Evolution (LTE) of 3G [1] and WiMAX [2].

The problem of joint optimum channel and power assignment to interfering sources is NP-hard, and a nonconvex mixed integer programming problem [3]. The only way to find the global optimum is exhaustive search, namely, for each possible channel assignment, the optimum power allocation has to be found. Therefore further simplification of the problem is required.

One approach is to allow each user to independently find its own allocation, while taking the interference it causes to others as a cost, which can be communicated between the neighbors. This idea has been used in many of the game theoretic resource allocation methods [4] [5]. However the solution the system reaches at equilibrium is unlikely to be the global optimum, and in fact may be far from it [6]. However, for ad hoc communication this approach might be the only option.

When the network has a known, fixed structure however, a better approach is to exploit this knowledge in order to simplify the problem. For example, for cellular networks this approach will be as follows. A predetermined Inter-Cell Interference Coordination (ICIC) rule can be found for the given network parameters, i.e., cell size, transmit powers, cell load, etc. The ICIC determines the inter-cell resource management [1]. Then each cell can independently schedule its users, for example using frame by frame Proportionally Fair (PF) scheduling [7], while following the inter-cell interference rules.

In this work we are proposing a two phase solution: the ICIC adaptation phase (i.e. Interference Concentration (ICon)) and intra-cell scheduling phase. At each iteration of ICon, inter-cell resource management is found, given the performance achieved in each cell in the previous iteration. Then, having fixed the the inter-cell resource management, intra-cell scheduling problem becomes a linear programming problem for which fast and simple solutions exist [8].

The idea behind the two phase approach is that when the inter-cell resource management rule is found and optimized using communication theory and experiments, the solution space of the original NP-hard problem is limited to the most reasonable options. And although global optimality is not guaranteed in this method either, it greatly reduces the problem size and complexity.

- We first propose ICon, a novel inter-cell resource management method based on defining Interference Power Profiles (IPPs) for cells. The IPP defines a limit to received interference on each sub-band, which the neighboring cells are obliged to meet. An example is given in Figure 1. The idea is that if the high interference causing users from all neighboring cells are concentrated on the same band, the bandwidth will be used more efficiently. 
- In order to balance performance across cells, ICon is made adaptable. At each ICIC adaptation time, cells broadcast the average utility they achieved in the previous period (e.g. over the X2 interface in LTE). Then each cell updates the IPP it imposes on its neighbors, given its performance relative to theirs. Each cell loosens its IPP requirements for neighbors that have worse performance than itself, and tightens it for neighbors that perform better.

- In the Intra-cell scheduling phase each cell finds the transmit power limits for each user on each sub-band such that none of its neighbors' IPPs is violated. This is easily done using channel gains between each user and the neighboring base stations (BSs), which is available at each user given pilots from neighboring BSs, assuming channel reciprocity holds (available in LTE for use in hand-off). Once the maximum transmit powers are found for each user on each sub-band, a linear optimization problem can be solved by each base station in order to allocate channels to its users, maximizing the minimum rate in the cell. This can be updated frequently since linear optimization problems are efficiently solved.

Our contributions in this work are twofold. First is that we propose a novel inter-cell interference management scheme based on interference concentration (i.e. ICon). ICon outperforms the common ICIC methods in cell edge user performance, it is simple to implement, easily adaptable, and can be applied to all OFDMA-based networks. Secondly, we combine ICon with inter-cell resource optimization and propose a two phase solution that adaptively improves the global performance of the network.

The rest of the paper is as follows. Related work will be presented in Section II. We define the optimization problem we aim to solve in Section III and propose our ICIC and intra-cell scheduling method. In Section IV we propose the adaptive ICIC method. The simulations are reported in Section V.

\section{RELATED WORK}

An example of a common ICIC method is Fractional Frequency Reuse (FFR) [9] [10]. In FFR, the bandwidth is partitioned into two sections each with a different reuse factor. Commonly one partition has frequency reuse of 1 and the rest reuse of 3 . The partition with reuse 1 is used for users in the inner circle of each cell which cause little interference to other cells. On the other hand the bordering users of each cell are assigned the reuse 3 bands, which are not used in any of the first tier neighbors of the cell. The performance of this method depends on the level of interference coupling between cells. In low coupling, FFR would only cause low spectral efficiency and not much gain compared with reuse 1 . Additionally, since part of the
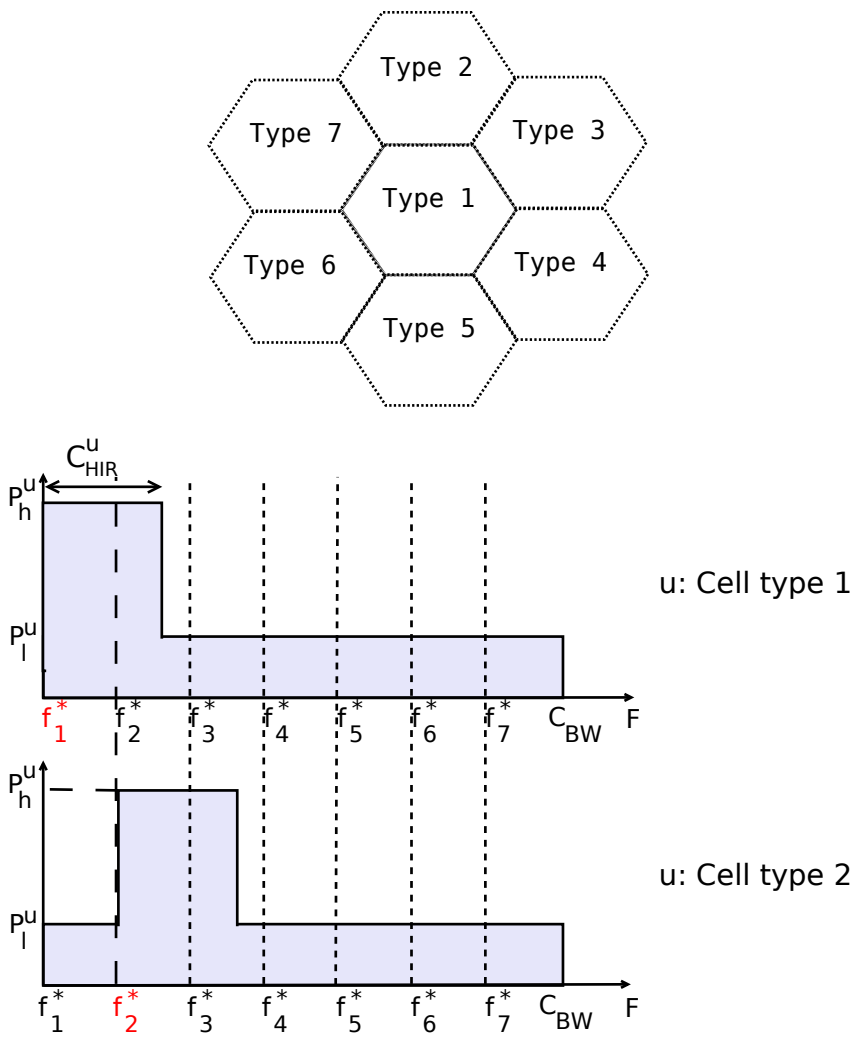

Fig. 1. An example of Interference Power Profiles (IPPs) set by ICon method for cell types 1 and 2 in a hexagonal cellular network. $\mathrm{f}_{i}^{*} \mathrm{~s}$ are the starting subbands for the High Interference Region (HIR) of each cell type. $C_{H I R}^{u}$ is the number of subbands in HIR, and can be any value between 0 and $C_{B W}$.

spectrum is used with reuse of 3, spectral efficiency is low and the peak rate is less than that of reuse 1 [11] [12] [13] [14].

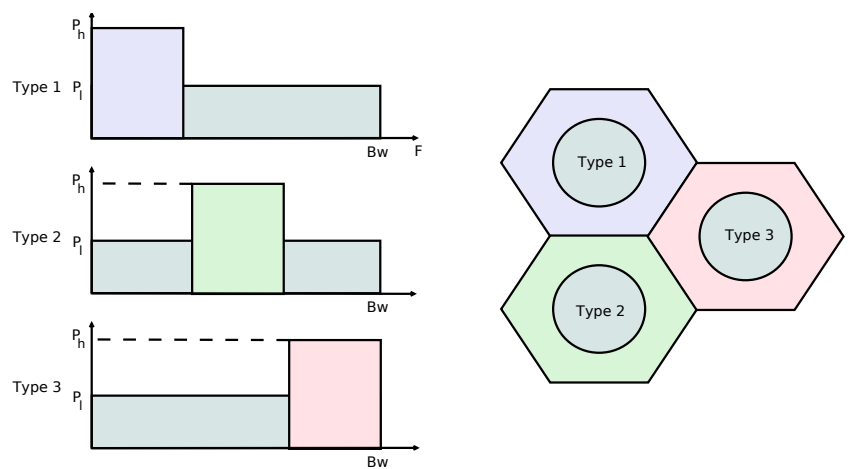

Fig. 2. Transmit power profiles for each cell type in a hexagonal cellular network, using SFR. The colored section of each cell only has access to the parts of the spectrum with the matching color, the inner sections of cells have access to the whole spectrum.

Another common ICIC method is Soft Frequency Reuse (SFR). In this method frequency is reused in every cell, however each cell is assigned a transmit power profile, with complementary patterns across neighboring cells, as shown in Figure 2 [15] [12]. 
An interesting ICIC method which was recently proposed is the inverted reuse scheme [16], [17]. In inverted reuse, users are restricted to a given transmit power profile, if they are found to be sufficiently close to a given cell. To find sufficiently close users, a threshold is used for the path loss difference between the serving BS and the neighboring BS. Since all users in all cells neighboring a cell will be given the same power profile, the received interference at the cell will be concentrated as given by that power profile. Our proposed ICIC scheme uses a similar idea as the inverted reuse, that is to shape the interference of a cell. However, we directly define an interference power profile (IPP) for each cell which allows for more direct and intuitive adaptation.

The work in [18] also uses the idea of assigning interference profiles for uplink ICIC using Overload Indicators (OIs) defined by $3 \mathrm{gpp}$ for LTE of 3G. Fixed interference profiles similar to transmit power profiles in SFR are assigned to cells. This work requires each cell to identify the high interference users in the nearby cells, and therefore requires more processing and inter-cell communication.

For inter-cell resource allocation, Proportionally Fair (PF) scheduling is a common method for LTE. This method is simple to implement and can be modified to achieve different levels of fairness [7]. Additionally for single cell OFDM networks, the optimal allocation of power per subband per user is found to be a convex optimization problem [3], solvable in polynomial time.

\section{OPTIMIZATION PROBLEM}

We consider a 2-D multi-cell network with OFDMA multiple access scheme. Users in each cell are allocated resources by the scheduler located in the cell base station for uplink communication. The resources to assign are power and channel allocation to each user. Each of the independent orthogonal channels are assumed to be Gaussian and interference is considered to be equivalent to noise in terms of channel capacity. The variables to find are each user's transmit power spectral density for each channel, $p_{i, c}$ and channel assignment, $a_{i, c}$ which is a binary array. We assume no intra cell interference. The following rate is achieved for user $i$ in cell $k$ :

$$
R_{i, k}=\sum_{c=1}^{C} a_{i, c} \cdot B_{c} \cdot \log _{2}\left(1+\frac{p_{i, c} \cdot g_{i k}}{N_{0} \cdot B_{c}+\sum_{u=1, u \neq k}^{K} P_{u k c}^{I}}\right),
$$

where $g_{i k}$ is the channel gain from user $i$ to receiver of cell $k, B_{c}$ is the bandwidth of channel $c . N_{0}$ is the noise power and $P_{u k c}^{I}$ is the received interference power from cell $u$ to cell $k$ on channel $c$. Namely, $P_{u k c}^{I}=\sum_{j \in X_{u}} a_{j, c} \cdot p_{j, c} . g_{j k}$.

$$
\begin{array}{llr}
\underset{\boldsymbol{a}, \boldsymbol{p}}{\operatorname{aximize}} & \min _{i, k}\left(R_{i, k}\right) & \\
& \sum_{i \in k} a_{i, c}=1, \quad a_{i, c} \in\{0,1\} \quad \forall k \\
& P_{M I N} \leq p_{i, k} \leq P_{M A X}, \quad \forall i, k
\end{array}
$$

where $R_{i, k}$ is given as Eq. (1). This problem is NP-hard for $(C>1)$ [3]. NP hardness of (2) means that optimality of any solution cannot be guaranteed unless with exhaustive search over all possible solutions. Therefore we can only use intuition and solutions that are experimentally proven to be effective to simplify the problem.

To do this, we first devise a new inter-cell interference coordination method, ICon, in order to decouple the inter-cell resource allocation problem from the intra-cell scheduling. The intra-cell scheduling problem will consist of assigning maximum allowed power to users without violating the IPPs set by ICon, and solving a linear optimization problem in order to assign subbands to users. ICon can then be adapted in order to increase the overall network utility.

\section{A. ICon}

Each cell is given an IPP which neighboring cells are required to respect, as shown in Figure 1. High Interference Region (HIR) is the section of frequency band that allows high levels of interference for each cell, as shown in the figure. We assume $P_{h}$ is set to allow maximum transmit power for bordering cells on the HIR. Depending on the type of each cell, the HIR starts at a given subband, $f_{i}^{*}$. There are two design parameters for the IPP,

$P_{l}^{u}$ : Interference power limit on low interference sub-bands

and

$$
C_{H I R}^{u}: \text { Number of sub-bands in HIR. }
$$

These parameters are assumed to be known if the network structure is known. Then, for each channel and each user, the maximum power that does not violate any of the neighbors' Interference Profile (IPP) should be found.

$$
p_{i, c}^{\max }=\min \left(\min _{u, u \neq k} \frac{I_{u}(c)}{g_{i u}}, P_{M A X}\right)
$$

where $I_{u}(c)$ is the value of IPP of cell $u$ at channel $c$, and $g_{i u}$ is the channel gain from user $i$ to base station of cell $u$. This can be found by the user from pilots from neighboring base stations, if we assume channel reciprocity, and can be transmitted to BS of cell $k$ (this information is also available in the UEs in cellular networks for handover).

The number of sub-bands in HIR, i.e. $C_{H I R}^{u}$, can be as high as $C_{B W}$, meaning that equal high interference is allowed on all bands, or can be very small, concentrating all the interference on a small band. Furthermore, since $C_{H I R}^{u}$ can 
be set individually for each cell (or modified adaptively as proposed in Section IV) our IPP can easily be adapted given the load in each cell. This is in contrast with FFR and SFR where the frequency divisions are generally not modifiable, although the load on each frequency section can be set individually in each cell. Additionally, ICon essentially merely imposes transmit power limits for users on different subbands based on their location in the cell, therefore resource allocation to users can be performed independently, unlike FFR and SFR where users are assigned to bands depending on their location.

\section{B. Channel and power assignment in each cell}

In this section we find the power assignment and channel allocation for cell $k$. In each cell the the scheduler is located at the base station. In the previous section, we fixed the maximum interference levels on each channel in each cell, given by the IPP of a cell. We now approximate Problem (2) by assuming $P_{u k c}^{I}$ to be constant during a scheduling period. This approximation results in Problem (2) becoming separable and solved in each cell. Then if $a_{i, c}$ is relaxed to be real valued, (i.e. the optimization problem will be solved every $\mathrm{T}$ frame lengths, and the $a_{i, c}$ will be rounded), Problem (2) becomes separable into the following optimization problems in each cell $k$ :

$$
\begin{aligned}
& \underset{\boldsymbol{a}, \boldsymbol{p}}{\operatorname{aximize}} t \\
& t \leq \sum_{c=1}^{C} a_{i, c} \cdot B_{c} \cdot \log _{2}\left(1+\frac{p_{i, c} \cdot g_{i k}}{N_{0} \cdot B_{c}+P_{k c}^{I}}\right) \quad \forall i \\
& \sum_{i \in k} a_{i, c}=T, \quad 0 \leq a_{i, c} \leq T \\
& P_{M I N} \leq p_{i, k} \leq p_{i, c}^{\max },
\end{aligned}
$$

where $P_{k c}^{I}$ is the total interference power received at cell $k$ at subband $c$. We can further simplify the problem by noting that the optimum transmit power for this modified problem is simply the maximum transmit power allowed for each user, i.e. $p_{i, c}^{*}=p_{i, c}^{\max }$. Then the problem becomes a linear programming problem in $\boldsymbol{a}$ :

$\underset{a}{\operatorname{Maximize}} t$

$$
\begin{aligned}
& t \leq \sum_{c=1}^{C} a_{i, c} \cdot B_{c} \cdot \log _{2}\left(1+\frac{p_{i, c}^{\max } \cdot g_{i k}}{N_{0} \cdot B_{c}+P_{k c}^{I}}\right) \quad \forall i \\
& \sum_{i \in k} a_{i, c}=T, \quad 0 \leq a_{i, c} \leq T
\end{aligned}
$$

which has efficient solutions [8]. In the simulations we solve the optimization problem once every 10 frames, i.e. $\mathrm{T}=10$.

There are two issues with problem (5). One is that since all cells are solving this problem separately, $P_{k c}^{I}$ is not going to be constant during the scheduling period. Second, is that even if the interference is constant in a scheduling period, optimizing the resources individually in each cell does not guarantee convergence to the global optimum.
To circumvent these issues we propose the following. One, we define average interference, $\bar{P}_{k c}^{I}$ as an average of the previous $N_{T}$ scheduling periods which updates as follows:

$$
\bar{P}_{k c}^{I}(t)=\left(\frac{1}{N_{T}}\right) \cdot P_{k c}^{I}(t)+\left(1-\frac{1}{N_{T}}\right) \cdot \bar{P}_{k c}^{I}(t-1)
$$

This will be slow changing in one scheduling period. We use this value instead of the instantaneous $P_{k c}^{I}(t)$ to estimate the achievable rate in the next scheduling period. Secondly, by setting an interference power profile (IPP) for each cell in the ICIC phase, we steer the problem towards better global solutions, although global optimality is not guaranteed.

\section{ADAPTATION OF ICON}

We define an IPP in each cell to impose on each of its neighbors, and initially set all equal to the IPP found for the given network structure for the particular cell type. Modifiable attributes of the internal IPP are as explained in Section III-A and shown in Figure 3.

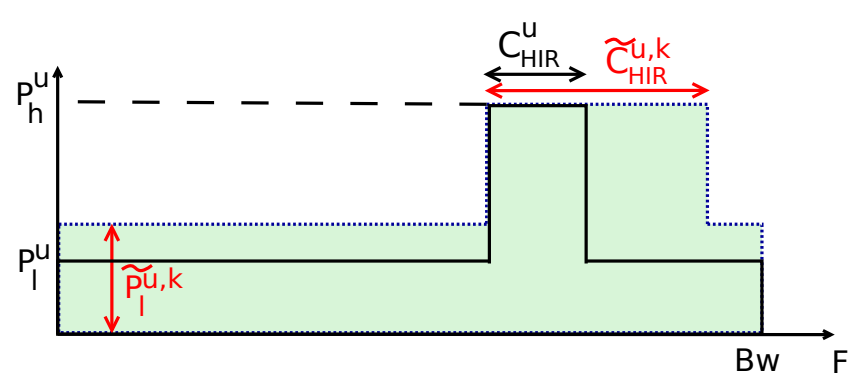

Fig. 3. Interference Power Profile (IPP) for cell $u$. Green curve is the IPP that cell $k$ has to respect for cell $u$.

At ICIC adaptation time, all cells broadcast the average utility they achieved in the previous period (e.g. on X2 channel in LTE). Then each cell $k$ updates the $C_{H I R}^{u}$ for the IPP it imposes on its neighbor $u$ as follows,

$$
\begin{aligned}
\widetilde{C}_{H I R}^{u, k}(t+1)= & \max \left\{\widetilde{C}_{H I R}^{u, k}(t)+\right. \\
& \left.\alpha \cdot\left(\frac{U^{u}(t)-U^{k}(t)}{\left(U^{u}(t)+U^{k}(t)\right) / 2}\right) \cdot C_{B w}, C_{H I R}^{u}\right\}
\end{aligned}
$$

and the low interference power limit, $\widetilde{P}_{l}^{u}$ is updated by,

$$
\begin{aligned}
\widetilde{P}_{l}^{u, k}(t+1)= & \max \left\{\widetilde{P}_{l}^{u, k}(t)+\right. \\
& \left.\beta \cdot\left(\frac{U^{u}(t)-U^{k}(t)}{\left(U^{u}(t)+U^{k}(t)\right) / 2}\right) \cdot P_{h}^{u}, P_{l}^{u}\right\}
\end{aligned}
$$

where $U^{k}(t)$ is the utility achieved in cell $k$ at time $t$. For our problem, $U^{k}(t)=\min _{i}\left(R_{i, k}\right) . \alpha$ and $\beta$ are step size values for the updates, and are in the range $[0,1]$. The larger the step size, the faster the convergence but the more the possibility of having to backtrack. Also, the larger step size value for a given parameter, the more adaptable that parameter will be. For example, a designer may choose to only adapt the $\widetilde{P}_{l}$ s and not the $\widetilde{C}_{H I R} \mathrm{~S}$, and vice versa by setting $\alpha$ or $\beta$ to zero. Step sizes can also be adaptive, and vary depending on the range 
TABLE I

SiMULATION PARAMETERS

\begin{tabular}{|l||c|}
\hline Parameter & Values \\
\hline Number of cells & 19, with wrap around \\
Users per cell for adaptive method & $19-21$, uniformly distributed \\
Site to site distance (m) & 130 \\
Bandwidth & $10 \mathrm{MHz}$ \\
Number of subbands & 63 \\
Max Power per user per band & $250 \mathrm{~mW} / 63$ \\
Path loss model (dB) & $30 \log _{10} R, \mathrm{R}$ in $(\mathrm{m})$. \\
Fractional power control & $\alpha=1, \Gamma$ differs. \\
IoT & ICon=10, Reuse $1=13(\mathrm{~dB})$ \\
PF scheduling parameters & $\mathrm{a}=3.5$ \\
FFR reuse 1 band /total band & $\eta=45 / 63$ \\
SFR parameters & $p_{l} / p_{h}=1 / 10, \gamma=6 \mathrm{~dB}$ \\
PF Scheduling at every: & 1 frame $(1 \mathrm{~ms})$ \\
Optimal Scheduling at every: & 10 frames $(10 \mathrm{~ms})$ \\
Step sizes for adaptive ICon & $\alpha=\beta=0.2$ \\
\hline
\end{tabular}

TABLE II

5 PERCENTILE RATE, ICON WITH PF, WITH IOT=10 DB IN LOW INTERFERENCE REGION.

\begin{tabular}{|c|c|c|c|c|}
\hline Rate(Kbps) & $P_{l}=P_{h} / 20$ & $P_{h} / 10$ & $P_{h} / 4$ & $P_{h} / 2$ \\
\hline$C_{H I R}=9$ & 429.50 & 487.00 & 568.00 & 491.00 \\
18 & 439.50 & 488.00 & 558.50 & 483.00 \\
27 & 458.50 & 535.00 & 545.50 & 496.50 \\
36 & 538.50 & $\underline{\mathbf{6 3 2 . 0 0}}$ & 539.00 & 476.00 \\
54 & 625.50 & 577.00 & 510.00 & 487.50 \\
\hline
\end{tabular}

of each parameter. Finally, the modified parameters need to be communicated to neighboring base-stations.

\section{Simulations}

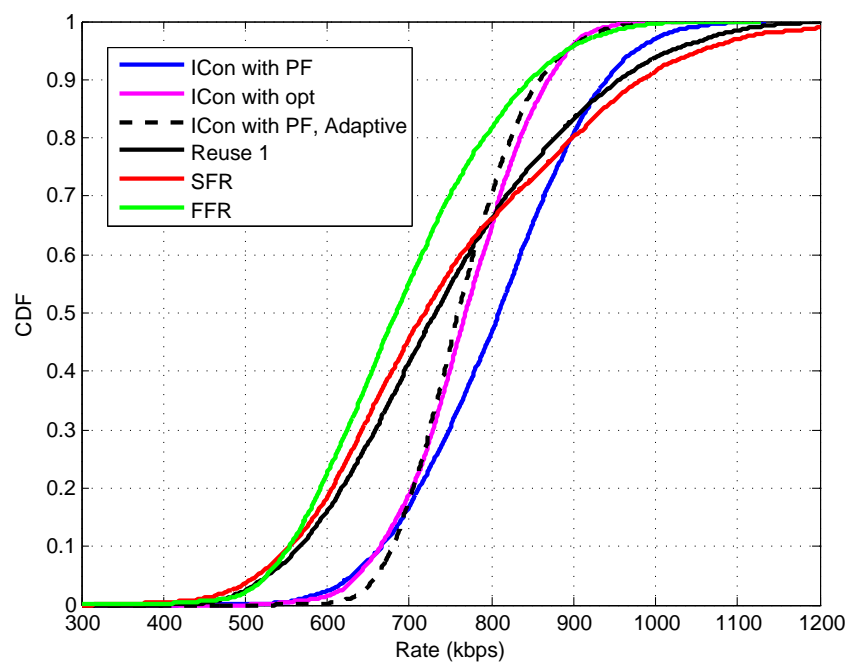

Fig. 4. CDF of achieved rates in a cell using various inter-cell interference coordination and scheduling schemes.

We provide Monte Carlo simulations for a 19 cell hexagonal 2-D cellular network, similar to one given in

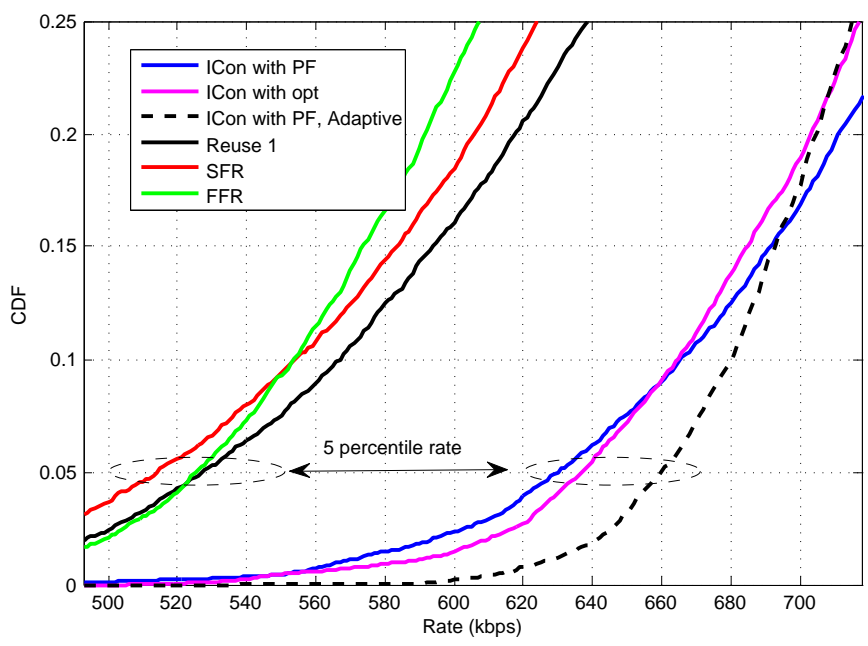

Fig. 5. Close-up of Fig. 4 demonstrating the five percentile rate achieved in each of the methods.

micro case for LTE [1]. We use wrap around in order to avoid boundary inconsistencies. Simulation parameters are given in Table I. We simulate several common ICIC and resource allocation methods: Reuse 1, SFR, and FFR, all with fractional power control and using proportionally fair scheduling, with parameters given in Table I. We simulate our ICIC method, ICon, with proportionally fair intra-cell scheduling, and with optimum intra-cell scheduling given in Section III-B. We perform simulations for 4 cell sizes, with site to site distance ranging from 32.5 (m) to 260 (m).

First, to find the $P_{l}$ and $C_{H I R}$ parameters for ICon, we find the 5 percentile rate achieved using a range of $P_{l}$ and $C_{H I R}$ values. An example is given in Table II, which demonstrates this for site to site distance of $130(\mathrm{~m})$. In this case $P_{l}=P_{h} / 10$ and $C_{H I R}=36$ achieves the highest 5 percentile rate.

Figure 4 demonstrates the CDFs of rates achieved by users in each scheme for site to site distance of $130(\mathrm{~m})$, and Figure 5 is the closeup of the 5 percentile region of the curves. Given these parameters, Reuse 1, SFR, and FFR achieve similar 5 percentile rates, but FFR performs worse than SFR and Reuse 1 for high rate users. ICon with both optimal and PF scheduling methods achieve higher 5 percentile rate than other methods, with $18 \%$ for ICon with PF and $20 \%$ for ICon with optimal scheduling. Additionally, we observe that the optimal scheduling does not achieve in a much higher performance compared with $\mathrm{PF}$, only about $1 \%$ gain in rate. The reason for this is that the approximation of interference in the next scheduling time as the average of the past is not accurate. Furthermore, optimal scheduling is done once every 10 frames, whereas PF is at every frame, which decreases the accuracy of interference prediction.

Lastly, we demonstrate the adaptation of ICon in Figure 6. Initially we run the simulations with static ICon with 
parameters as above, until the rates settle to fixed values. Then we start the adaptation algorithm. We plot the five percentile rate versus the number of iterations of the adaptive method. From the first iteration, the 5 percentile rate is instantly increased by $18 \mathrm{Kbps}$, and eventually converges to an $5 \%$ gain over static ICon. This demonstrates both rapid convergence and performance gain over the static case.

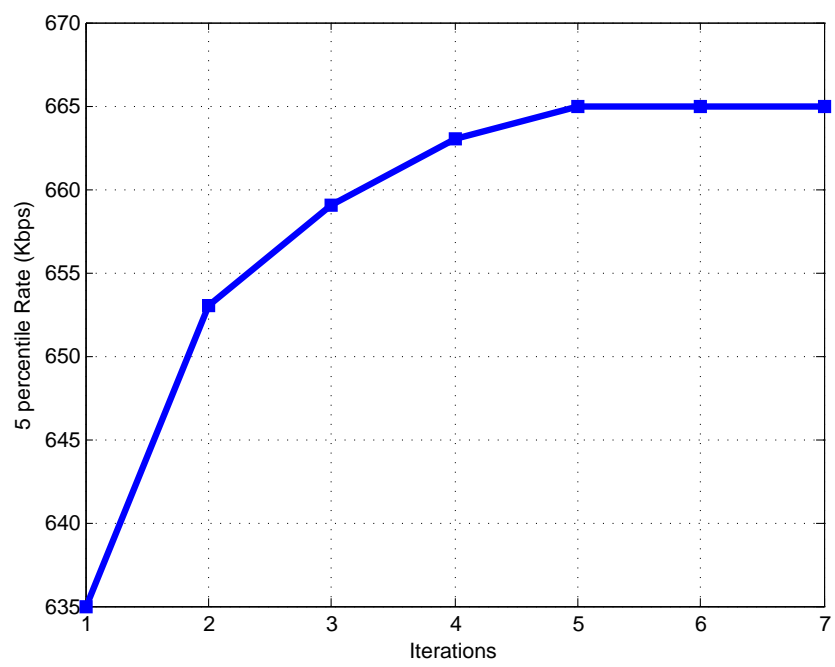

Fig. 6. Evolution of 5 percentile rate when the ICon adaptation is allowed. ICon with PF scheduling for site to site $=130(\mathrm{~m})$, users per cell: $19-21$, uniformly distributed.

\section{CONCLUSION}

In this work we propose a new inter-cell interference management method (ICon) for OFDMA based networks, such as LTE of $3 \mathrm{G}$ and WiMAX. ICon is based on setting interference power limits on each subband for each cell, requiring the neighboring cells to respect the imposed limits. Our simulations show that ICon achieves up to $18 \%$ gain over reuse 1 for the 5 percentile rate in a typical cellular network. ICon is easily and rapidly adapted to the performance obtained by each cell, which results in balancing of the performance over the whole network.

We also propose a two phase solution to the NP-hard problem of resource allocation in multicell OFDMA-based networks. First, ICon performs inter-cell resource management, updating interference power limits for each cell. Second, the intra-cell optimal scheduling assigns resources in each cell while meeting the constraints imposed by ICon. The optimal intra-cell scheduling is found to be a linear optimization problem for which efficient solutions exist. Static ICon with optimal scheduling performs $20 \%$ better than reuse 1 in terms of 5 percentile rate, and the adaptive method adds an additional $5 \%$ gain over this value.

\section{REFERENCES}

[1] 3GPP, "Physical Layer Aspects for Evolved UTRA," TR 25.814, V1.2.2 2006.

[2] 802.16e 2005, "Part 16: air interface for fixed and mobile broadband wireless access systems," Feb. 2006.
[3] Zhi-Quan Luo and Shuzhong Zhang, "Dynamic spectrum management: Complexity and duality," Selected Topics in Signal Processing, IEEE Journal of, vol. 2, no. 1, pp. 57 -73, Feb. 2008.

[4] Zhu Han, Zhu Ji, and K. Ray Liu, "Power minimization for multicell OFDM networks using distributed non-cooperative game approach," IEEE Global Telecommunications Conference, 2004. GLOBECOM '04., pp. 3742-3747, 2004.

[5] Jianwei Huang, Randall Berry, and Michael L Honig, "Distributed Interference Compensation for Wireless Networks," IEEE JOURNAL ON SELECTED AREAS IN COMMUNICATIONS, vol. 24, no. 5, pp. 1074-1084, 2006.

[6] Shunsuke Hayashi and Zhi-Quan Luo, "Spectrum Management for Interference-Limited Multiuser Communication Systems," IEEE Transactions on Information Theory, vol. 55, no. 3, pp. 1153-1175, Mar. 2009.

[7] C. Wengerter, J. Ohlhorst, and a. Golitschek Edler von Elbwart, "Fairness and Throughput Analysis for Generalized Proportional Fair Frequency Scheduling in OFDMA," 2005 IEEE 61st Vehicular Technology Conference, vol. 2, no. 2, pp. 1903-1907, 2005.

[8] S. Boyd and L. Vandenberghe, Convex Optimization, Cambridge Univ Press, Cambridge, U.K., 2004.

[9] Samuel W Halpern, "Reuse partitioning in cellular systems," in Vehicular Technology Conference, 1983, pp. 322 - 327.

[10] 3rd Generation Partership Project 2 (3GPP2), "Cdma2000 high rate packet data air interface specification revision a. technical report c.s20024-a," March 2004.

[11] Arne Simonsson, "Frequency Reuse and Intercell Interference CoOrdination In E-UTRA," 2007 IEEE 65th Vehicular Technology Conference - VTC2007-Spring, pp. 3091-3095, Apr. 2007.

[12] S-E. Elayoubi, O. Ben Haddada, and B. Fourestie, "Performance evaluation of frequency planning schemes in OFDMA-based networks," IEEE Transactions on Wireless Communications, vol. 7, no. 5, pp. 16231633, May 2008.

[13] Thomas Novlan, Jeffrey G Andrews, Illsoo Sohn, and Radha Krishna Ganti, "Comparison of Fractional Frequency Reuse Approaches in the OFDMA Cellular Downlink," in GLOBECOM, 2010.

[14] Balaji Rengarajan, Alexander L. Stolyar, and Harish Viswanathan, "Self-organizing Dynamic Fractional Frequency Reuse on the uplink of OFDMA systems," 2010 44th Annual Conference on Information Sciences and Systems (CISS), pp. 1-6, Mar. 2010.

[15] Huawei, "Soft frequency reuse scheme for UTRAN LTE," R1-050507, 3GPP TSG RAN WG1 2005.

[16] Christian G Gerlach, Ingo Karla, Andreas Weber, Lutz Ewe, Hajo Bakker, Edgar Kuehn, and Anil Rao, "ICIC in DL and UL With Network Distributed and Self-Organized Resource Assignment Algorithms in LTE," Bell Labs Technical Journal, vol. 15, pp. 43-62, 2010.

[17] Nageen Himayat, Shilpa Talwar, Anil Rao, and Robert Soni, "Interference Management for 4G Cellular Standards," IEEE Communications Magazine, , no. August, pp. 86-92, 2010.

[18] Minghai Feng, Xiaoming She, and Lan Chen, "Coordinated Scheduling based on Overload Indicator for LTE / LTE-A Uplink," in Vehicular Technology Conference, 2010. 